\title{
THE SUPERCRITICAL PILE GAMMA-RAY BURST MODEL: THE GRB AFTERGLOW STEEP DECLINE AND PLATEAU PHASE
}

\author{
J. Sultana ${ }^{1}$, D. Kazanas ${ }^{2}$, And A. Mastichiadis ${ }^{3}$ \\ ${ }^{1}$ Mathematics Department, Faculty of Science, University of Malta, Msida MSD2080, Malta; joseph.sultana@um.edu.mt \\ 2 Astrophysics Science Division, NASA/Goddard Space Flight Center, Greenbelt, MD 20771, USA \\ ${ }^{3}$ Department of Physics, University of Athens, Panepistimiopolis, GR 15783 Zografos, Greece \\ Received 2013 March 31; accepted 2013 August 26; published 2013 November 19
}

\begin{abstract}
We present a process that accounts for the steep decline and plateau phase of the Swift X-Ray Telescope (XRT) light curves, vexing features of gamma-ray burst (GRB) phenomenology. This process is an integral part of the "supercritical pile" GRB model, proposed a few years ago to account for the conversion of the GRB kinetic energy into radiation with a spectral peak at $E_{\mathrm{pk}} \sim m_{e} c^{2}$. We compute the evolution of the relativistic blast wave (RBW) Lorentz factor $\Gamma$ to show that the radiation-reaction force due to the GRB emission can produce an abrupt, small $(\sim 25 \%)$ decrease in $\Gamma$ at a radius that is smaller (depending on conditions) than the deceleration radius $R_{D}$. Because of this reduction, the kinematic criticality criterion of the "supercritical pile" is no longer fulfilled. Transfer of the proton energy into electrons ceases and the GRB enters abruptly the afterglow phase at a luminosity smaller by $\sim m_{p} / m_{e}$ than that of the prompt emission. If the radius at which this slow-down occurs is significantly smaller than $R_{D}$, the RBW internal energy continues to drive the RBW expansion at a constant (new) $\Gamma$ and its X-ray luminosity remains constant until $R_{D}$ is reached, at which point it resumes its more conventional decay, thereby completing the "unexpected" XRT light curve phase. If this transition occurs at $R \simeq R_{D}$, the steep decline is followed by a flux decrease instead of a "plateau," consistent with the conventional afterglow declines. Besides providing an account of these peculiarities, the model suggests that the afterglow phase may in fact begin before the RBW reaches $R \simeq R_{D}$, thus providing novel insights into GRB phenomenology.
\end{abstract}

Key words: cosmological parameters - gamma-ray burst: general

Online-only material: color figures

\section{INTRODUCTION}

Gamma-ray bursts (GRBs) are extremely bright explosions at cosmological distances (Costa et al. 1997; van Paradijs et al. 1997) with isotropic luminosities occasionally exceeding $\sim 10^{54} \mathrm{erg} \mathrm{s}^{-1}$. Their durations are in the range $\sim 0.1-1000 \mathrm{~s}$ and their luminosities peak at an energy close to the electron rest mass energy, $E_{\mathrm{pk}} \sim 1 \mathrm{MeV}$ (however, the accumulation of observational data has shown that this characteristic energy exhibits a wider distribution, ranging from as low as a few $\mathrm{keV}$ (Campana et al. 2006) to as high as $15 \mathrm{MeV}$ (Axelsson et al. 2012), in correlation with either the isotropic energy released in the burst, $E_{\text {iso }}$, or its peak isotropic luminosity $L_{\mathrm{p} \text {,iso }}$ ).

They are believed to originate in the collapse of stellar cores (long GRBs) or the mergers of neutron stars (short GRBs), processes that result in jet-like relativistic outflows with Lorentz factors $\Gamma \lesssim 300$ (but on occasion exceeding values 500-1000; Abdo et al. 2009a, 2009b; Ackermann et al. 2010; Hascoët et al. 2012). It is generally considered that the kinetic energy of these outflows is converted efficiently into radiation in collisions of shells of matter ejected at different times by the GRB "central engine"; such collisions are thought to be necessary in order to produce the observed rapid GRB variability (see, however, Narayan \& Kumar 2009) and spectra with characteristic GRB signatures, i.e., a maximum luminosity at energies $E_{\mathrm{pk}} \simeq m_{e} c^{2}$ (for reviews, see Piran 2004 for bursts prior to the launch of Swift and Zhang 2007 for bursts after the Swift launch). Following this most luminous, prompt, $\gamma$-ray emission phase, GRBs shift into their afterglow phase. In this phase, their luminosity is substantially lower and their peak emission shifts into the X-ray band. The longer duration of this phase $\left(\Delta t \sim 10^{5} \mathrm{~s}\right)$ allows a more precise localization and optical detection, which can then provide the GRB redshift.

According to prevailing theory (Piran 2004; Zhang 2007), GRB emission is due to synchrotron radiation by electrons accelerated, in the prompt phase, in the shocks of the colliding shells. In the afterglow, the emission is due to electrons accelerated in the forward shock of the expanding relativistic blast wave (RBW). As the RBW expands, it sweeps more matter. After it has swept up an amount $M \simeq E / c^{2} \Gamma^{2}$ ( $E$ is its total injected energy, $\Gamma$ its asymptotic Lorentz factor) at a distance $R_{D}$, its Lorentz factor decreases and it is thought to enter the afterglow phase, as surmised by its declining X-ray and optical fluxes. Under these assumptions, one can calculate the expected $\mathrm{X}$-ray flux decrease with time, which turns out to be a power law, $F_{X} \propto t^{-\alpha}$, with $\alpha \simeq 1$ in spherical (Sari et al. 1998) and $\alpha \simeq 2$ in jet-like (Sari et al. 1999) flows. Indeed, the early, sparsely sampled, pre-Swift light curves appeared consistent with such a behavior. However, more densely sampled X-ray light curves with the X-Ray Telescope (XRT) aboard Swift uncovered significant deviations from this behavior. So, following the prompt Swift Burst Alert Telescope (BAT) $\gamma$-ray emission, typical XRT afterglows (Tagliaferri et al. 2005; Nousek et al. 2006; Evans et al. 2009) comprise a segment of much steeper flux decline $\left(\propto t^{-3}\right.$ to $\left.t^{-6}\right)$, followed by either a less steep power law (Liang et al. 2007), or a $10^{2}-10^{5} \mathrm{~s}$ period of nearly constant flux (a "plateau"), followed finally at $t=T_{\text {brk }}$ by the more conventional power-law decline $\simeq t^{-1}$. In addition, Swift follow-ups also discovered (Burrows et al. 2005) occasional flares on top of these light curves, as late as $\sim 10^{5} \mathrm{~s}$ after the BAT trigger. These unexpected details in the GRB afterglow light curves were added to the other already open problems related to the GRB prompt 
emission, namely the nature of their "inner engine," the nondissipative transport of their energy to the emission region to distances $R \sim 10^{16}-10^{17} \mathrm{~cm}$ and its efficient dissipation there, and the physics behind the distribution of the GRB peak energy $E_{\mathrm{pk}} \sim 1 \mathrm{MeV}$ (Mallozzi et al. 1995; Preece et al. 2000).

These prompt GRB emission issues are usually settled by fiat in most studies, e.g., by assuming that a large fraction $(\sim 50 \%)$ of the proton post-shock energy is converted into electrons with a minimum Lorentz factor $\gamma_{\mathrm{min}}$, chosen so such that the burst luminosity maximum would appear at $E_{\mathrm{pk}}$. However, this exhausts essentially most of the models' freedom, setting the afterglow evolution on the path described in Sari et al. (1998), thus making an account of the observed afterglow light curve shapes all the more pressing. There have been a number of attempts to account for at least some of these features. Thus, Kazanas et al. (2007) suggested that if the post-shock proton distribution function comprises, in addition to a relativistic Maxwellian of $T \sim \Gamma m_{p} c^{2}$, also a power law that extends to energies $\gamma m_{p} \gg \Gamma m_{p}$, one could account for the steep decline followed by the more conventional $\propto t^{-1}$ power-law decline light curves of GRB afterglows. A similar proposal was put forward by Giannios \& Spitkovsky (2009), who employed the realistic electron distributions produced in particle-in-cell (PIC) simulations, which do have a form similar to that conjectured in Kazanas et al. (2007). More recently, Petropoulou et al. (2011) provided models with the desired general afterglow shape by adjusting the maximum electron distribution Lorentz factor $\gamma_{\max }$ in such a way that the steep decline represents synchrotron emission by electrons near $\gamma_{\max }$ (which are cooling fast), while attributing the constant X-ray flux component to inverse Compton emission by the (much more slowly varying) lower energy section of the electron distribution. Another commonly accepted interpretation of the steep decay phase in the GRB early afterglow light curves is that the high-latitude emission, i.e., the curvature effect (Zhang 2006 and references therein), follows the prompt emission phase. The goal of the present work is to show that the XRT afterglow light curves can be accounted for by incorporating the supercritical pile model (hereafter SPM) and its feedback on the dynamics of the GRB RBWs that gives rise to the GRB.

The SPM (Kazanas et al. 2002; Mastichiadis \& Kazanas 2006, 2009) has been introduced to address the issue of the GRB dissipation and the apparently efficient conversion of the RBW kinetic energy into radiation with the observed spectral characteristics. The fundamental process of this model is a radiative instability that can convert the internal energy of the RBW relativistic protons into relativistic $e^{+}, e^{-}$pairs. The conversion takes place on timescales $\Delta R / c(\Delta R$ is the typical width of the RBW) via the $p \gamma \rightarrow p e^{+} e^{-}$reaction, provided that certain kinematic and dynamic threshold (criticality) conditions are fulfilled, which are discussed in the next section. Unlike the more conventional GRB models in the literature, the SPM does not require (but does not forbid) accelerated particle populations besides those produced by the isotropization of the RBW kinetic energy behind the shock. Most importantly, it does not invoke an ad hoc equipartition between the proton and electron energy densities behind the shock, as it includes the dynamics that convert the proton energy into $e^{+}, e^{-}$. Finally, a consequence of the kinematic threshold condition of the $p \gamma \rightarrow p e^{+} e^{-}$reaction is the natural emergence (after all relevant Lorentz transformations) of a characteristic photon peak energy, $E_{\mathrm{pk}} \simeq 1 \mathrm{MeV}$ (in the observer frame, assuming that the process operates close to its kinematic threshold), largely independent of the
RBW Lorentz factor and in agreement with observations (e.g., Mallozzi et al. 1995; Goldstein et al. 2012). In more conventional models, such a characteristic photon energy occurs only at the expense of assuming the presence of a low energy cut-off in the electron distribution function.

More recently, Mastichiadis \& Kazanas (2009) explored numerically the SPM from the prompt into the afterglow stage by computing the evolution and spectrum of a RBW of Lorentz factor $\Gamma_{0}=100$ propagating through a medium of density $n(r) \propto r^{-2}$, representing the wind of a Wolf-Rayet star. In this treatment, they incorporated the effects of the radiative drag into the RBW evolution equations, where these effects are introduced in the production of the GRB spectra by the bulk Comptonization of upstream scattered photons, a crucial element of this model. They showed that when both threshold conditions of the model were fulfilled and the energy stored in protons is converted into radiation, the resulting radiation reaction force reduced the RBW Lorentz factor $\Gamma$ and also the GRB flux. In the particular case they examined, the drop in $\Gamma$ was sufficiently large to render the RBW subcritical. The GRB thus entered the afterglow phase (with its luminosity coming only from the electrons being swept up by the RBW) after only a couple of seconds (in the observer's frame), thus producing a short GRB, even though the RBW was assumed to propagate through a pre-supernova stellar wind medium.

In this work, we employ a simplified version of the RBW evolution discussed in Mastichiadis \& Kazanas (2009) in order to focus our attention to the entire evolution of $\Gamma$ from its acceleration phase into its late time decline. Using this simplified version, we provide an account of the vexing steep decline and plateau phase in the early GRB afterglow light curves observed in most long GRBs. The simplified version of this approach allows us to compute the evolution of both conical and parabolic GRB jet configurations in a medium of constant density $\rho$ from their accelerating phase (i.e., $\Gamma \simeq 1$ ) to their adiabatic decay past the deceleration radius. In Section 2, we introduce the general framework of the SPM and criticality conditions. In Section 3, we present our results and then in Section 4 we summarize our findings and present our conclusions.

\section{THE SUPERCRITICAL PILE MODEL IN BRIEF}

The process described in this section was first used in the context of active galactic nuclei (AGNs) by Kazanas \& Mastichiadis (1999), who employed it to argue for the possibility of a hadronic origin of the relativistic electrons in blazars. It involves the combination of: (1) The relativistic proton plasma radiative instability of Kirk \& Mastichiadis (1992) as applied to a RBW. (2) The increase in the energy of synchrotron photons produced in this plasma, which, upon their scattering in upstream-located matter (referred to as the "mirror"), are then re-intercepted by the RBW, as discussed in Ghisellini \& Madau (1996). The instability of Kirk \& Mastichiadis (1992) is basically that of a nuclear pile: synchrotron photons, produced by $e^{+}, e^{-}$pairs, interact with the relativistic protons of the plasma to produce more $e^{+}, e^{-}$pairs; the set-up is radiatively unstable if the column of the plasma is sufficiently large such that at least one of the $N$ photons $\left(N \simeq \gamma / b \gamma^{2}=1 / b \gamma ; \gamma\right.$ is the electron Lorentz factor and $b$ the magnetic field normalized to the critical one $B_{c}=m_{e}^{2} c^{3} / e \hbar \simeq 4.4 \times 10^{13} \mathrm{G}$ ) produced by a member of an $e^{+}, e^{-}$pair of energy $E_{e} \simeq \gamma m_{e} c^{2}$ produces another pair in reaction with a relativistic proton before escaping the system by the process $p \gamma \rightarrow p e^{-} e^{+}$. 
If $R$ is the size of the plasma and $n_{0}$ is the proton density, this last constraint reads $\sigma_{p \gamma} R n_{0} \gtrsim 1 / N \simeq b \gamma$. However, the $p \gamma$ process requires that the energy of the synchrotron photon $E_{s}$ be sufficiently high to produce a pair in the proton rest frame, i.e., $\gamma E_{s} \gtrsim 2 m_{e} c^{2}$. Considering that $E_{s} \simeq b \gamma^{2} m_{e} c^{2}$, the kinematic threshold reads $\gamma^{3} \gtrsim 2 / b$. Incorporating this in the column density constraint, one obtains $\sigma R n_{0} \gamma^{2} \gtrsim 2$. Applying these considerations to the particles in the post-shock region of a RBW, one can set their $\gamma$ values equal to the RBW Lorentz factor $\Gamma$ so that the criticality conditions are expressed in terms of the bulk RBW kinematic properties, i.e., $\Gamma^{3} \gtrsim 2 / b$ and $\sigma_{p \gamma} R n_{0} \Gamma^{2} \gtrsim 2$.

If the synchrotron photons scatter upstream of the RBW in a "mirror" (in AGNs, these mirrors are broad-line region clouds) of scattering depth $\tau_{\text {mirr }}$, upon their re-interception by the RBW they have energies larger by $4 \Gamma^{2}$. This modifies the kinematics and also the column density conditions to

$$
\Gamma^{5} \gtrsim(1 / 2 b) \text { and } 2 \tau_{\text {mirr }} n_{0} \sigma R \Gamma^{4} \geqslant 1 .
$$

With the above setting for the conversion of proton energy into electrons and photons, it was shown in Kazanas et al. (2002) that the threshold of the $p \gamma \rightarrow p e^{-} e^{+}$reaction translates into an energy $b \Gamma^{5}$ in the observer's frame, which, by the first of the relations above, implies a peak emission energy at roughly the electron rest mass, in agreement with observations.

The observed peak energy occurs at this value only if the process operates close to the kinematic threshold at all times. The fact that $b \Gamma^{5} \sim 1 / 2$ does not suffice to produce a burst because, while $b \Gamma^{5}$ may be well above the threshold, say $10 \mathrm{MeV}$ or higher, rapid proton energy release requires also that the column of swept-up protons be sufficiently high such that the dynamic threshold in Equation (1) also be satisfied. Apparently, the larger $b \Gamma^{5}$ is, the faster the accumulated energy will be released once supercritical. Therefore, the model does not exclude the higher values of $E_{\mathrm{pk}}$ observed recently (Axelsson et al. 2012; Guiriec et al. 2013). More importantly, the bright bursts analyzed in these references indicate a correlation between the value of $E_{\mathrm{pk}}$ and the burst luminosity ( $L \propto E_{\mathrm{pk}}^{\alpha}, \alpha \simeq 1.3$ ) during the evolution of the same burst (not addressed to the best of our knowledge by models to date), suggesting additional nuances; it would be unwise to speculate at this point.

On the other hand, values of $E_{\mathrm{pk}}$ smaller than $1 \mathrm{MeV}$ (Campana et al. 2006) averaged over the burst duration may be due to larger viewing angles of the GRB jet $\theta>1 / \Gamma$, as discussed in Ioka \& Nakamura (2001). Also, $E_{\mathrm{pk}}<1$ can be obtained for $\theta<1 / \Gamma$ if the shock produces, in addition to protons with energy $\sim \Gamma m_{p} c^{2}$, a power-law tail that extends to energies $\gamma \gg \gamma_{1}>\Gamma$ such that $b \gamma_{1}^{5} \gtrsim 1 / 2$, while $b \Gamma^{5}<1 / 2$, as discussed in Kazanas et al. (2007). Independently of the specifics discussed above, the important point to bear in mind is the emergence of a characteristic energy in agreement with observations after all Lorentz transformations have taken place, as a result of the physics of the dissipation process.

The evolution of $\Gamma$ of a RBW is given by the coupled mass and energy-momentum conservation laws (see, e.g., Chiang \& Dermer 1999). In the case that the RBW plows through a radiation field, one must also include the effects of the radiation reaction of the RBW as it plows through the radiation that has scattered upstream of the shock (Mastichiadis \& Kazanas 2008; Boettcher \& Principe 2009); these effects are given below:

$$
\frac{d M}{d R}=4 \pi R^{2} \Gamma \rho-\frac{\dot{E}}{c^{3} \Gamma}
$$

and

$$
\frac{d \Gamma}{d R}=\Gamma \frac{A R_{0} / R^{2}}{1+A R_{0} / R}-\frac{4 \pi R^{2} \rho \Gamma^{2}}{M}-\frac{F_{\mathrm{rad}}}{M c^{2}},
$$

where $R(t)$ is the radius measured from the center of the original explosion and $A=E_{0} / M_{0} c^{2}$, where $E_{0}$ and $M_{0}$ represent the initial total energy and rest mass, respectively, of the flow at $R=R_{0}$, corresponding to the radius of the GRB progenitor. Here, $\dot{E}$ represents the radiation emission rate as measured in the comoving frame and $F_{\text {rad }}$ is the radiation reaction force exerted on the RBW by the radiation field exterior to the flow, given by

$$
F_{\text {rad }}=\frac{64 \pi}{9 c} \tau_{b} n_{e} \sigma_{T} R \Gamma^{4} \dot{E},
$$

where $\tau_{b}$ is the RBW Thomson depth, $\sigma_{T}$ is the Thomson cross section, and $n_{e}$ is the circumstellar matter electron density assumed to be the same as the proton density $n_{0}$ used in Equation (1).

Mastichiadis \& Kazanas (2009) applied these equations to compute both the evolution of the Lorentz factor $\Gamma$ and the emitted radiation for a RBW propagating through the wind of a Wolf-Rayet star, i.e., a medium with density profile $n(R) \propto R^{-2}$ that presumably was the progenitor of the supernova that gave rise to the RBW. The values of $\dot{E}$ and $F_{\text {rad }}$ were computed by implementing the numerical code, originally described in Mastichiadis \& Kazanas (2006), to solve the equations

$$
\frac{\partial n_{i}}{\partial t}+L_{i}+Q_{i}=0
$$

where the functions $n_{i}$ represent the differential number densities of protons, electrons, and photons with the index $i$ taking any of the subscripts $p, e$ or $\gamma$, while $L_{i}$ denotes the losses and escape and $Q_{i}$ denotes the injection and source terms in the system.

The detailed calculations of Mastichiadis \& Kazanas (2009), using the parameters $R_{0}=10^{14} \mathrm{~cm}, n_{0}=8 \times 10^{8} \mathrm{~cm}^{-3}, \Gamma_{0}=$ $100, B_{0}=4.4 \times 10^{4} \mathrm{G}$, and $E_{0}=10^{54} \mathrm{erg}$ different from those used herein-except for $E_{0}$ - but appropriate for the setting considered, confirmed the qualitative estimates concerning the positions and effects of the problem thresholds. In addition, they showed that the radiation reaction effects were significant, and had an immediate effect on $\Gamma$, which slowed down over a length scale short compared with $R_{0}$ to a value lower than that required by the kinematic threshold of the problem. This resulted in the precipitous decline of the GRB luminosity as the only available energy to be radiated from that point on was that of the sweptup electrons. At the same time, they computed the resulting spectrum and found it to be in agreement with the basic premises of the SPM (i.e., exhibiting a peak at $E_{\mathrm{pk}} \sim m_{e} c^{2}$ ). The resulting spectrum also agreed with the spectrum softening significantly with the decrease in $\Gamma$ effected by the radiation reaction process, with the GRB thus entering the afterglow stage.

\section{RESULTS}

In the present paper, we study a simplified version of the evolution of the RBW Lorentz factor $\Gamma$ in a medium of constant number density $n_{0}$ within the SPM. To this end, we begin our computations at the radius of the GRB progenitor, $R_{0}$, where we set $\Gamma_{0}=1$, with approximate estimates for the resulting luminosity, a fact that allows a much broader search in parameter space and exploration of the evolution over longer time scales. The evolution of $\Gamma$ is followed from its initial accelerating phase to its saturation (constant $\Gamma$ ) and slow-down stages, attributing 
each to the prompt or afterglow stage depending on whether the criticality conditions are fulfilled.

To reduce the number of free parameters, we assume that the magnetic field is in equipartition with the post-shock pressure so that $B \simeq 0.4\left(n_{0} / 1 \mathrm{~cm}^{-3}\right)^{1 / 2} \Gamma \mathrm{G}$. Then, the long-term evolution of the Lorentz factor depends on the free parameters $E_{0}, R_{0}$, and $n_{0}$, which determine the radius where the kinematic and dynamic conditions are satisfied so that the RBW becomes supercritical. It could happen that for certain parameter combinations the threshold conditions are satisfied at more than one radius, in which case the released energy should be proportional to the time between such bursts, as found by Ramirez-Ruiz \& Merloni (2001). We examine both conical and parabolic configurations of the GRB jet with parameters $R_{0}=10^{11} \mathrm{~cm}, n_{0}=100 \mathrm{~cm}^{-3}$, $M_{0} c^{2}=5 \times 10^{51} \mathrm{erg}$, and total isotropic energy $E_{0}=10^{54} \mathrm{erg}$.

\subsection{Conical Outflows}

The evolution of $\Gamma$ is given by the solution of the coupled Equations (2) and (3). To simplify our treatment, instead of using the numerical code employed in Mastichiadis \& Kazanas (2006) and Mastichiadis \& Kazanas (2009) to calculate the radiation emission rate $\dot{E}$, we use the fact that once the criticality conditions are satisfied almost all the energy in the swept-up protons is immediately radiated away, so that

$$
\dot{E}=\dot{E}_{\text {inj }}=4 \pi R^{2} \rho\left(\Gamma^{2}-\Gamma\right) c^{3},
$$

where $\dot{E}_{\text {inj }}$ is the proton energy injection rate (Blandford \& McKee 1976). Letting $x=R / R_{0}$, the evolution equations in Equations (2) and (3) become

$$
\frac{d M}{d x}=4 \pi R_{0}^{3} x^{2} \Gamma \rho-4 \pi x^{2} R_{0}^{3} \rho(\Gamma-1)
$$

and

$$
\begin{aligned}
\frac{d \Gamma}{d x}= & \frac{\Gamma A}{x(x+A)}-\frac{4 \pi x^{2} R_{0}^{3} \rho \Gamma^{2}}{M} \\
& -\frac{256 \pi^{2}}{9 M} \tau_{b} n_{e} \sigma_{T} x^{3} R_{0}^{4} \rho \Gamma^{4}\left(\Gamma^{2}-\Gamma\right) .
\end{aligned}
$$

As long as the threshold conditions in Equation (1) are not satisfied, then the evolution of the RBW is described approximately by Equations (7) and (8) without the $\dot{E}$ and $F_{\text {rad }}$ terms on the right-hand side. This is the standard non-radiative case mentioned earlier in which the Lorentz factor reaches its asymptotic value $\Gamma \simeq A=E_{0} / M_{0} c^{2}=200$ and then proceeds with the conventional decline of afterglow theory, shown by the dashed curve in Figure 1. For the chosen values of the GRB parameters, the kinematic condition is satisfied at $\log \left(R / R_{0}\right) \simeq 2.77$ (represented by the first vertical red line in Figure 1) before the Lorentz factor reaches even its asymptotic value. Once enough matter is piled up such that the column of accumulated hot protons exceeds its critical value, the RBW becomes supercritical at $\log \left(R / R_{0}\right) \simeq 4.55$ (represented by the green vertical line in Figure 1); the proton accumulated energy is released on the shock light crossing time scale and results in a sudden drop in $\Gamma$ of the RBW due to radiative drag. The decrease in $\Gamma$ reduces the value of $b \Gamma^{5}$ below its threshold value at $\log \left(R / R_{0}\right) \simeq 4.56$ (represented by the second red line in Figure 1) and arrests the conversion of proton energy into radiation. The luminosity drops precipitously, by roughly a factor $m_{p} / m_{e}\left(m_{p}\right.$ and $m_{e}$ are the proton and electron masses, respectively) as the emitted radiation now comes only from the cooling of the electrons

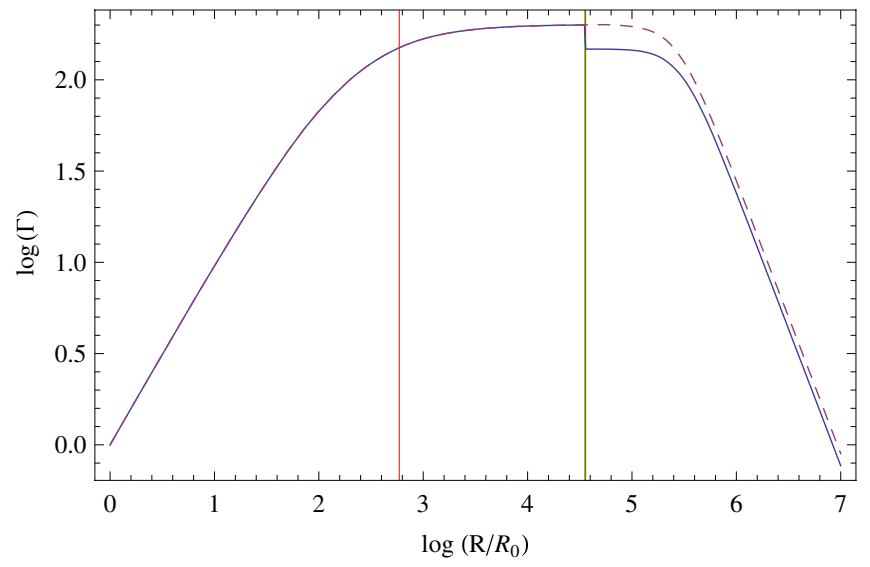

Figure 1. Lorentz factor for a RBW propagating in a constant density environment for a conical GRB outflow. The dashed curve represents the evolution with radius in the adiabatic case without radiative drag. The red vertical lines represent the region where the kinematic condition is satisfied and the green line indicates the radius at which the dynamic condition starts to be satisfied. The GRB parameters are given in the text.

(A color version of this figure is available in the online journal.)

swept by the RBW and the GRB enters the afterglow stage (one should note here that, in contrast with most models, the SPM provides natural, physical grounds for the separation of GRBs in the prompt and afterglow stages).

However, despite the decrease in luminosity that follows the reduction in $\Gamma$ due to the effects of radiation reaction, the rest mass accumulated to this point may be too small, for the given RBW internal energy $E_{0}$, to produce a decrease in $\Gamma$ in the manner expected generally for distances $R>R_{D}$. Therefore, the RBW evolution must continue at a constant (or even increasing) $\Gamma$, even though, according to the premises of the SPM, the GRB has entered the afterglow stage (the kinematic threshold condition is not satisfied; the $p \gamma \rightarrow p e^{+} e^{-}$reaction does not take place and hence $\left.E_{\mathrm{pk}} \ll m_{e} c^{2}\right)$. The value of $\Gamma$ will remain constant at a distance equal to the deceleration radius $R_{D}$, as shown in Figure 1. We contend that this stage is responsible for the plateau phase observed in the XRT light curves.

In Figure 3 (left) and (right), we plot the evolution of two RBWs with the same initial conditions as those of Figures 1 and 2, except for the value of $M_{0} c^{2}$, which now determines the asymptotic value of $\Gamma_{\infty}$ in the absence of radiation reaction. The value of $M_{0} c^{2}$ for these features is set to $M_{0} c^{2}=3.5 \times 10^{51} \mathrm{erg}$ and $M_{0} c^{2}=2 \times 10^{51} \mathrm{erg}$ for Figure 3 (left) and (right), respectively, implying asymptotic $\Gamma$ values of $\simeq 286$ and 500 . For these conditions, the radiation reaction feedback is much larger, with the sharp transition taking place at a distance increasingly closer to $R_{D}$ with increasing value of $\Gamma_{\infty}$. This then implies a concomitant decrease in the length of the constant $\Gamma$ section in the afterglow stage, the latter effectively disappearing for the largest value of $\Gamma_{\infty}$. This is of interest because of the correlation between the plateau luminosity and the time $T_{\mathrm{bk}}$ of its break to the more conventional afterglow decrease with time (Dainotti et al. 2008, 2010). It is worth noting that the evolution of $\Gamma$ in Figure 3 (right) would likely correspond to one of the typical shapes of the afterglow curves indicated in Willingale \& O'Brien (2007), i.e., that of a steep decline, followed by a conventional time decrease of the XRT flux.

\subsection{Parabolic Outflows}

In a parabolic outflow, the accumulated number of ambient particles increases as $N(R) \propto R^{2}$, unlike the conical case 


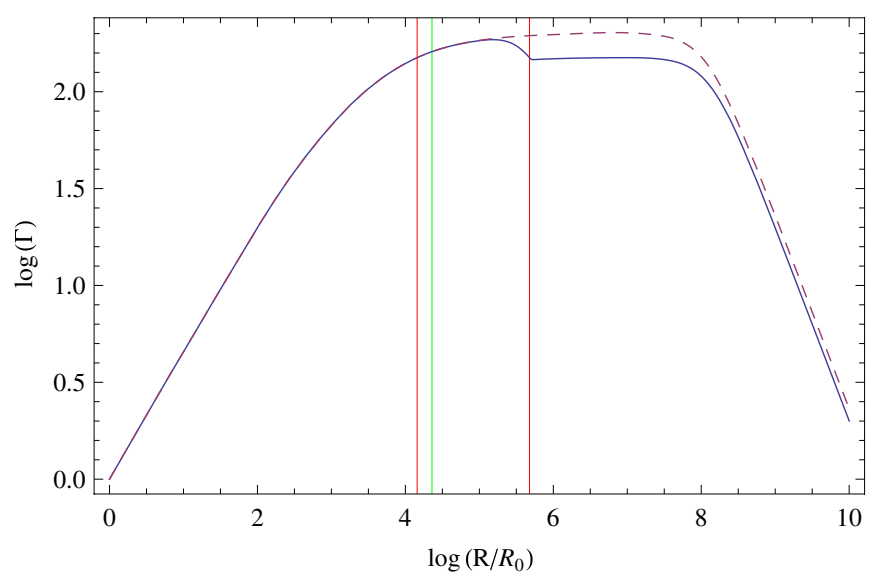

Figure 2. Lorentz factor for a RBW propagating in a constant density environment for a parabolic GRB outflow. The dashed curve represents the evolution with radius in the adiabatic case without radiative drag. The red vertical lines represent the region where the kinematic condition is satisfied and the green line indicates the radius at which the dynamic condition starts to be satisfied. The GRB parameters are given in the text.

(A color version of this figure is available in the online journal.)

where $N(R) \propto R^{3}$. Therefore, the evolution equations with the previous assumption that $\dot{E}=\dot{E}_{\text {inj }}$ are given by

$$
\frac{d M}{d x}=4 \pi R_{0}^{3} x \Gamma \rho-4 \pi x R_{0}^{3} \rho(\Gamma-1)
$$

and

$$
\begin{aligned}
\frac{d \Gamma}{d x}= & \frac{2 \Gamma A}{3 x\left(x^{2 / 3}+A\right)}-\frac{4 \pi x R_{0}^{3} \rho \Gamma^{2}}{M} \\
& -\frac{256 \pi^{2}}{9 M} \tau_{b} n_{e} \sigma_{T} x^{2} R_{0}^{4} \rho \Gamma^{4}\left(\Gamma^{2}-\Gamma\right) .
\end{aligned}
$$

Solving these differential equations numerically in a constant density medium with the same GRB parameters used previously in the conical case gives the evolution of the Lorentz factor $\Gamma$ with radius $R$, as shown Figure 2. Again, the dotted curve represents the evolution in the adiabatic case without radiative drag. In the parabolic configuration, the expanding RBW accumulates mass at a lower rate and therefore has a larger deceleration radius $R_{D}$ than the conical case; for the same reason, the luminosity released is smaller. In fact, both threshold conditions are satisfied during the acceleration phase of the RBW when the first term on the right-hand side of Equation (10) is dominant, i.e., before the RBW Lorentz factor reaches its asymptotic value $\Gamma=200$. These factors explain the delay and smoothness of the drop in $\Gamma$ when the RBW becomes supercritical.

As can also be seen in Figure 2, the slowing down of the RBW due to radiative drag during the supercritical phase reduces $b \Gamma^{5}$ below its threshold value, thereby ending the prompt GRB phase. This is followed by a period of constant $\Gamma$, which is longer than that obtained previously due to the fact that the RBW becomes supercritical during its accelerated expansion phase and the fact that, for a given $E_{0}$, it sweeps up matter at a lower rate than a conical RBW. After all the non-adiabatic effects have died down and once the RBW has accumulated enough mass, the evolution of $\Gamma$ beyond the deceleration radius follows the conventional decay $\Gamma(R) \propto R^{-1}$ appropriate for a parabolic flow.

\subsection{The X-Ray Flux}

The fact that the Lorentz factor remains constant for a period does not guarantee that the corresponding X-ray flux does too. Such an outcome depends also on the particular process responsible for the X-ray emission. For example, if the RBW propagates in a medium of constant density (as assumed herein), with the magnetic field in equipartition with the plasma (i.e., $\left.B \simeq 0.4\left(n_{0} / 1 \mathrm{~cm}^{-3}\right)^{1 / 2} \Gamma \mathrm{G}\right)$, the observed flux would increase with time because the source specific intensity would remain the same (it sweeps the same amount of electrons per unit time) while its solid angle (its size) increases. Therefore, the constant XRT emission during the plateau stage imposes certain restrictions on the emission process. If the observed X-ray emission is due to bulk Comptonization, just like the $\gamma$-ray prompt emission, then the $R^{-2}$ decrease of the ambient photons could indeed offset the $\propto R^{2}$ increase of the RBW surface to produce a constant $\mathrm{X}$-ray flux. An alternative is that the plateau $\mathrm{X}$-ray emission is due to synchrotron emission but by a magnetic field that decreases with radius like $B(R) \propto R^{-1}$, since the emissivity is proportional to $B(R)^{2} \propto R^{-2}$, thereby offsetting again the increase in the RBW area. Finally, if the ambient particle density decreases like $R^{-2}$, i.e., the RBW propagates in
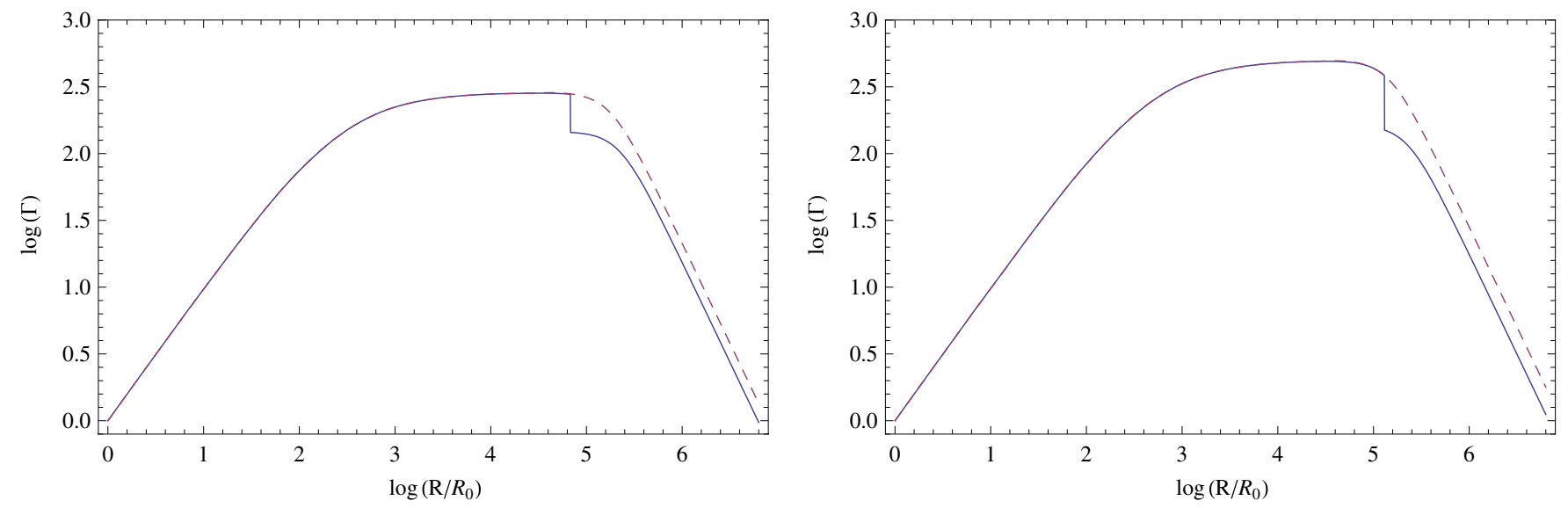

Figure 3. Lorentz factor evolution of two conical RBWs propagating in the constant density environment of Figure 1. These are distinguished from that of Figure 1 only by their initial rest mass energies, which are $M_{0} c^{2}=3.5 \times 10^{51}$ and $2.0 \times 10^{51}$ erg for the left and right figures, respectively. The dashed curves represent the evolution with radius in the adiabatic case without radiative drag. The remaining GRB parameters are given in the text.

(A color version of this figure is available in the online journal.) 


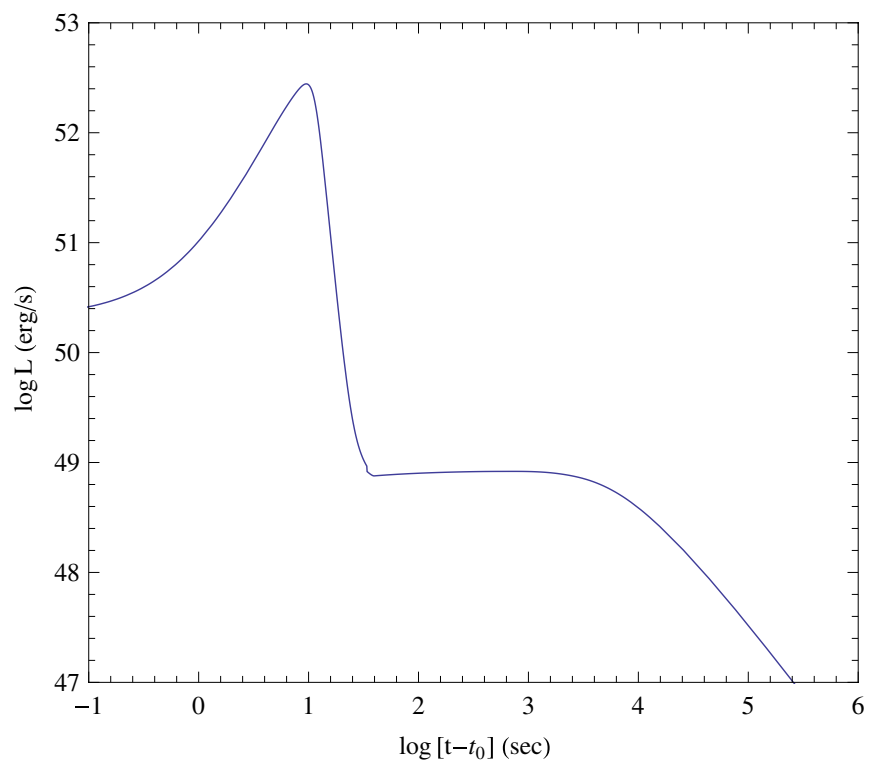

Figure 4. Bolometric luminosity of a RBW propagating in a constant density environment for a parabolic GRB outflow. The rising part is the prompt BAT emission, while the steep and plateau parts are the XRT afterglow, one assuming that the density of the photons scattered in the "mirror" decreases as $R^{-2}$. The time $t_{0}$ represents the time of the onset of the burst.

(A color version of this figure is available in the online journal.)

a stellar wind environment, that would also lead to a constant flux (Shen \& Matzner 2012); however, the present calculations for the evolution of $\Gamma$ would have to be revised to reflect the different density dependence on $R$.

The situation is not too different in the parabolic expansion case, provided that the observer "sees" only a fraction of the expanding RBW front. In Figure 4, we show the bolometric light curve of a GRB with the kinematic characteristics of the parabolic outflow shown in Figure 2, assuming that the emission past the radiative reaction slow down is due to bulk Comptonization, i.e., that the number of photons decreases with radius as $R^{-2}$. One has to bear in mind, however, that this is only a toy model light curve, based on a number of simplifying assumptions. However, this, along with the dynamics of radiation reaction feedback, set the stage for a more detailed future exploration of these issues.

\section{SUMMARY AND DISCUSSION}

In the present work, we have studied the evolution of the Lorentz factor $\Gamma$ of a GRB RBW from its origin $\left(\Gamma_{0} \sim 1\right.$ at $R=$ $R_{0}$ ) through its acceleration ( $\Gamma \propto R$ for conical outflows and $\Gamma \propto R^{2 / 3}$ for parabolic outflows) to saturation $(\Gamma=$ constant $)$ and decay phases, within the context of the GRB SPM. We have argued within the framework of well-understood, defined physical processes and without the introduction of a posteriori assumptions that this evolution provides, among others: (1) A definition of what constitutes the prompt GRB phase, its broader spectral features, and a criterion for its termination and the onset of the afterglow. (2) An account of the steep decline and plateau or the steep decline and power law phase of the GRB afterglow, observed in the largest number of afterglows (Evans et al. 2009) and their relation to the prompt emission properties.

Of these, (1) has been discussed in several of our previous publications (Kazanas et al. 2002; Mastichiadis \& Kazanas 2006, 2009), where it was shown that the SPM can provide both an efficient, rapid conversion of the energy stored in relativistic protons in the RBW of a GRB into electrons while at the same time producing a spectrum with $E_{\mathrm{pk}} \sim m_{e} c^{2}$, in agreement with observations. In the present work, this process has been placed within the broader context of an evolving RBW and it is shown that the prompt GRB phase lasts as long as this efficient transfer of energy from protons into electrons is allowed by the kinematics of the pair production process, which depend crucially on $\Gamma$. With the reduction of $\Gamma$ below the critical value set by the $p \gamma \rightarrow p e^{+} e^{-}$reaction threshold, this transfer stops and the GRB luminosity decreases precipitously along with the value of $E_{\mathrm{pk}}$.

Point (2) is the novel aspect of the present work that shows that incorporating the radiation reaction associated with the flowing of the RBW through its upstream scattered radiation (or, for that matter, any sufficiently intense ambient radiation) in the evolution of $\Gamma$ can produce a small, sharp, (over a distance $\Delta R \lesssim R$ ) but important decrease of the RBW Lorentz factor value $\Gamma$. Although small, this decrease is important because it pushes the value of $\Gamma$ below that of the kinematic threshold of the SPM (at least within the confines of the simplified treatment of radiation emission used herein). As a result, the transfer of energy from protons into electrons ceases and leads to a steep reduction in the GRB luminosity by roughly a factor $\sim m_{p} / m_{e}$, consistent with the decrease in luminosity between the prompt and afterglow GRB luminosities. In Figure 5 (left) and (right), we present a sample of two such XRT light curves, namely those of GRB 110420A and GRB 120213A, taken from Nat Butler's compilation (http://butler.lab.asu.edu/swift/older.html). In these figures we note with the thick yellow arrow a range of $\simeq 2000$ in flux to provide a visual estimate of the change in flux between the prompt and afterglow stages, which indeed is consistent with the estimate given here. We have also estimated by inspection the same ratio in a number of other bursts in the same list; we found some of them to be smaller and some larger. Smaller values come from in cases where not all protons are "burnt," as happens to be the case in the numerical simulations of this process. Larger values of the ratio will result from a significant decrease in $\Gamma$, considering that the observed luminosity is proportional to $\sim \Gamma^{4}$. Such is the case in Figure 5 (left) and (right), which produce values $\Gamma^{4} \simeq 10$ and 100 , respectively. A more detailed statistical analysis of this issue is currently under consideration.

As long as the RBW radius at the point of this transition is smaller than its deceleration radius $R_{D}$, following this decrease in $\Gamma$ (and $L$ ) due to the radiation reaction process, the internal energy to rest mass ratio of the RBW is sufficiently high to ensure that its Lorentz factor $\Gamma$ remains constant until its radius reaches the value $R_{D}$. At this point, $\Gamma$ begins its more conventional decline. We have also argued that during this period of constant $\Gamma$, the value of the X-ray flux can remain roughly constant, in agreement with observations, provided that certain conditions are fulfilled (described in Section 3.3). Interestingly, synchrotron emission from a RBW propagating into a medium of constant density and magnetic field would yield a flux increasing with time and it is excluded in most cases. The effect of radiation reaction feedback was considered in Mastichiadis \& Kazanas (2008) and in more detail in Mastichiadis \& Kazanas (2009). However, the conditions of the Mastichiadis \& Kazanas (2009) study were such that the radiation reaction transition radius was close to $R_{D}$, just like that of Figure 3 (right), so the constant $\Gamma$ section was not discernible (nonetheless, the steep and then more shallow decline of the $10 \mathrm{keV}$ flux was apparent in their Figure 2 (right)). 

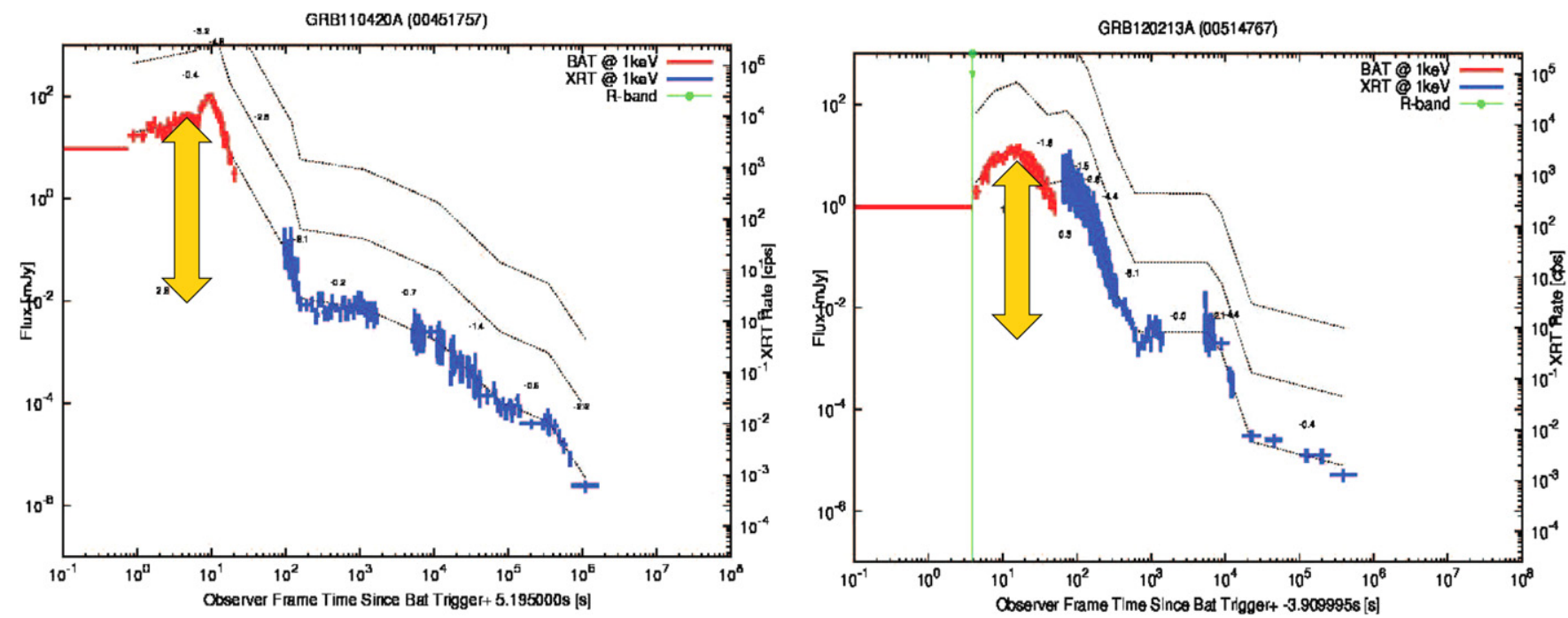

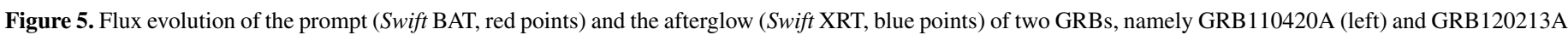

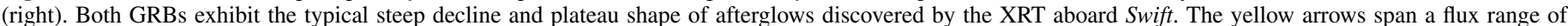
approximately $\simeq m_{p} / m_{e}$, the value implied by the physics of the SPM.

(A color version of this figure is available in the online journal.)

Viewed more broadly, Figures 1 and 3 (left) and (right) present a set of calculations of the evolution of the Lorentz factor of three different RBWs with the same initial conditions of radius $\left(R_{0}=10^{11} \mathrm{~cm}\right)$, internal energy $\left(E_{0}=10^{54} \mathrm{erg}\right)$, and background (uniform) density $\left(n=100 \mathrm{~cm}^{-3}\right)$, but with different values of their initial internal rest mass energy $M_{0} c^{2}$, namely $M_{0} c^{2}=5 \times 10^{51}, 3.5 \times 10^{51}$, and $2 \times 10^{51} \mathrm{erg}$, respectively. The following values of asymptotic Lorentz factor, $\Gamma_{\infty}$ corresponds: 200,286 , and 500, respectively. These values are indeed achieved and when both thresholds of the SPM are satisfied, the proton energy is released to produce the main GRB emission and, under the force of the radiation reaction, the Lorentz factor is reduced sharply to $\Gamma \simeq 160$ in all three cases. However, the duration of the plateau in the evolution of $\Gamma$ becomes shorter with decreasing $M_{0} c^{2}$. This is an important fact in view of the systematics between the plateau X-ray luminosity and its duration $T_{\text {brk }}$ (Dainotti et al. 2008, 2010). The goal of the present paper is not to resolve this issue but to point out that the considerations discussed herein, which associate $R_{D}$ with $T_{\mathrm{bk}}$ rather than with the onset of the afterglow, provide a novel framework within which such issues can be discussed and perhaps resolved. Furthermore, since emission both prior to and after the radiation reaction reduction in $\Gamma$ takes place while $\Gamma$ was approximately constant, it may not be surprising to find that the GRB properties in these two stages are correlated, even though one stage belongs to the prompt epoch and the other belongs to the afterglow GRB stage. This is indeed the correlation found by Sultana et al. (2012).

One of the most challenging issues of the SPM, raised a number of times at conferences and indeed by the referee of this paper, is that of the fast variability, $\Delta t \ll t$, of the GRB prompt emission, a prominent characteristic of the majority of GRBs. This poses a problem, considering that the propagation of a blast wave in a uniform medium cannot produce variability times shorter than $R / c \Gamma^{2}$. Considering small, spherical inhomogeneities in the swept-up medium was shown to be very inefficient (Sari \& Piran 1997). The broadly accepted solution, as of this writing, is that of "internal shocks" (Rees \& Mészáros 1994), i.e., variations in the activity of the GRB central engine that result in different sections of the outflow catching up with each other and colliding to produce the observed variability. However, Narayan \& Kumar (2009) argued that this process is only moderately efficient $(\sim 1 \%-10 \%)$; at the same time, they noted that model fits to the data of specific GRBs produce prompt emission locations much larger than those inferred from the colliding shock locations (as does the SPM). For these reasons, these authors opted for relativistic turbulence to provide additional boosting to small regions within the broader blast wave, indicating that this notion could resolve the GRB fast variability issue. On the other hand, numerical simulations by Zrake \& McFadyen (2013) have shown that such turbulence would quickly dissipate in the absence of a continuous energy input. It is possible that such an input is provided by the reconnection of the turbulently amplified magnetic field in the post-shock region, as suggested by Zhang \& Yan (2011), who presented a detailed investigation of GRB variability based on the notion of magnetic field reconnection.

One could also speculate that these processes may be possible to integrate within the SPM, a process that is beyond the scope of the present work. Clearly, a uniform medium cannot produce the observed variability and a collection of "blobs" in the ambient medium can only be efficient under the conditions discussed in Narayan \& Kumar (2009). Nonetheless, recent PIC simulations (Silva et al. 2003; Nishikawa et al. 2006; RamirezRuiz et al. 2007) have shown that relativistic shocks such as those from GRB RBWs are unstable to filamentation via Weibel (Weibel 1959) or two-stream (Buneman 1958) instabilities. If the column density of the RBW is $\Sigma$, the splitting of the flow by these instabilities into, say, $N$ filaments, will increase their local column density to $\sim N \Sigma$ (there will likely be a distribution of columns, each becoming supercritical at different times); since the crucial quantity for the SPM is the local column, filamentation makes the conversion of RBW kinetic energy into radiation all the more efficient, with each such filament playing the role of the turbulent eddies in the model of Narayan \& Kumar (2009). Of course, each such filament independently produces (via the SPM process) relativistic electrons that plow through the ambient radiation to produce the observed emission, which is perceived as a single pulse in the overall GRB prompt emission. Admittedly, these PIC simulations probe only scales 
associated with their microscopic plasma physics quantities; however, Silva et al. (2003) do contend that these filaments may eventually organize into much larger scales (of the order of the shock width in our case). Whether these can reproduce the observed GRB variability is, at present, an open issue. The duration of the shots resulting from each such filament should then depend on the local electron cooling time.

In summary, we note that the SPM, when integrated within the entire evolution of a GRB blast wave, provides, to the best of our knowledge, the first consolidation of the broader temporal and spectral properties of both the prompt and afterglow GRB stages within the framework of a single model. Considering that this model involves essentially no free a priori assumptions, it should not be expected to account for specific features of specific bursts; rather, it should be considered as a broader framework within which one attempts to account for the more specific GRB systematics. In this respect, we find it extremely encouraging that the same physics that provide for the efficient conversion of blast wave kinetic energy into radiation and the value(s) of $E_{\mathrm{pk}}$ also provide an account of the vexing XRT light curves. Furthermore, it makes a prediction for the bolometric luminosity prior to and after the radiation reaction reduction in $\Gamma$, indicating that this should be of order $m_{p} / m_{e}$. Indeed, a cursory search through the combined BAT-XRT light curves suggests that this is indeed the case; we are currently involved in providing a more complete statistic of the above statement, which will appear in a future publication.

We thank the anonymous referee for incisive, constructive comments that have added to the completeness of this work. J.S. gratefully acknowledges financial support from the University of Malta during his visit at the NASA-GSFC and the hospitality of the Astrophysics Science Division of the GSFC. D.K. acknowledges support from Swift and Fermi GO grants.

\section{REFERENCES}

Abdo, A. A., Ackermann, M., Ajello, M., et al. 2009a, ApJL, 706, L138 Abdo, A. A., Ackermann, M., Arimoto, M., et al. 2009b, Sci, 323, 1688 Ackermann, M., Asano, K., Atwood, W. B., et al. 2010, ApJ, 716, 1178 Axelsson, M., Baldini, L., Barbiellini, G., et al. 2012, ApJL, 757, L31 Blandford, R. D., \& McKee, C. 1976, PhFl, 19, 1130 Boettcher, M., \& Principe, D. 2009, ApJ, 692, 1374

Buneman, O. 1958, PhRvL, 1, 8
Burrows, D. N., Romano, P., Falcone, A., et al. 2005, Sci, 309, 1833

Campana, S., Mangano, V., Blustin, A. J., et al. 2006, Natur, 442, 1008

Chiang, J., \& Dermer, C. D. 1999, ApJ, 512, 699

Costa, E., Frontera, F., Heise, J., et al. 1997, Natur, 387, 783

Dainotti, M. G., Cardone, V. F., \& Capozziello, S. 2008, MNRAS, 391, L79

Dainotti, M. G., Willingale, R., Capozziello, S., Cardone, V. F., \& Ostrowski, M. 2010, ApJL, 722, L215

Evans, P. A., Beardmore, A. P., Page, K. L., et al. 2009, MNRAS, 397, 1177

Ghisellini, G., \& Madau, P. 1996, MNRAS, 280, 67

Giannios, D., \& Spitkovsky, A. 2009, MNRAS, 400, 330

Goldstein, A., Burgess, J. M., Preece, R. D., et al. 2012, ApJS, 199, 19

Guiriec, S., Daigne, F., Hascoët, R., et al. 2013, ApJ, 770, 32

Hascoët, R., Daigne, F., Mochkovitch, R., \& Vennin, V. 2012, MNRAS, 421,525

Ioka, K., \& Nakamura, T. 2001, ApJL, 554, L163

Kazanas, D., Georganopoulos, M., \& Mastichiadis, A. 2002, ApJL, 578, L15

Kazanas, D., \& Mastichiadis, A. 1999, ApJL, 518, L17

Kazanas, D., Mastichiadis, A., \& Georganopoulos, M. 2007, in Proc. of the VI INTEGRAL Workshop, The Obscured Universe, ed. S. Grebenev, R. Sunyaev, \& C. Winkler (ESA SP-622; Noordwijk: ESA), 577

Kirk, J. G., \& Mastichiadis, A. 1992, Natur, 360, 135

Liang, E. W., Zhang, B. B., \& Zhang, B. 2007, ApJ, 670, 565

Mallozzi, R. S., Paciesas, W. S., Pendleton, G. N., et al. 1995, ApJ, 454, 597

Mastichiadis, A., \& Kazanas, D. 2006, ApJ, 645, 416

Mastichiadis, A., \& Kazanas, D. 2008, in Proc. 30th Int. Cosmic Ray Conf., Vol. 3, ed. R. Caballero, J. C. D’Olivo, G. Medina-Tanco, L. Nellen, F. A. Sanchez, \& J. F. Valdes-Galicia, 1175

Mastichiadis, A., \& Kazanas, D. 2009, ApJL, 694, L54

Narayan, R., \& Kumar, P. 2009, MNRAS, 395, 472

Nishikawa, K. I., Hardee, P. E., \& Hededal, C. B. 2006, ApJ, 642, 1267

Nousek, J. A., Kouveliotou, C., Grupe, D., et al. 2006, ApJ, 642, 389

Petropoulou, M., Mastichiadis, A., \& Piran, T. 2011, A\&A, 531, A76

Piran, T. 2004, RvMP, 76, 1143

Preece, R. D., Briggs, M. S., Mallozzi, R. S., et al. 2000, ApJS, 126, 19

Ramirez-Ruiz, E., \& Merloni, A. 2001, MNRAS, 320, L25

Ramirez-Ruiz, E., Nishikawa, K. I., \& Hededal, C. B. 2007, ApJ, 671, 1877

Rees, M. J., \& Mészáros, P. 1994, ApJL, 430, L93

Sari, R., \& Piran, T. 1997, ApJ, 485, 270

Sari, R., Piran, T., \& Halpern, J. P. 1999, ApJL, 519, L17

Sari, R., Piran, T., \& Narayan, R. 1998, ApJL, 497, L17

Shen, R., \& Matzner, C. D. 2012, ApJ, 744, 36

Silva, L. O., Fonseca, R. A., Tonge, J. W., et al. 2003, ApJL, 596, L121

Sultana, J., Kazanas, D., \& Fukumura, K. 2012, ApJ, 758, 32

Tagliaferri, G., Goad, M., Chincarini, G., et al. 2005, Natur, 436, 985

van Paradijs, J., Groot, P. J., Galama, T., et al. 1997, Natur, 386, 686

Weibel, E. S. 1959, PhRvL, 2, 83

Willingale, R., \& O'Brien, P. T. 2007, RSPTA, 365, 1179

Zhang, B. 2006, ApJ, 642, 354

Zhang, B. 2007, ChJAA, 7, 1

Zhang, B., \& Yan, H. 2011, ApJ, 726, 90

Zrake, J., \& McFadyen, A. I. 2013, ApJL, 763, L12 